\section{Development of Simple Sequence Repeat Markers for Chionanthus retusus (Oleaceae) and Effective Discrimination of Closely Related Taxa}

\author{
Renée S. Arias \\ USDA-ARS Genomics and Bioinformatics Research Unit, Stoneville, MS \\ 38776
}

\author{
Natascha Techen \\ University of Mississippi, National Center for Natural Products Research, \\ School of Pharmacy, University, MS 38677
}

Timothy A. Rinehart

USDA-ARS Southern Horticultural Laboratory, Poplarville, MS 39470

Richard T. Olsen

U.S. National Arboretum, USDA-ARS, Washington, DC 20002

Joseph H. Kirkbride, Jr.

U.S. National Arboretum, USDA-ARS, Washington, DC 20002

\section{Brian E. Scheffler ${ }^{1}$ \\ USDA-ARS Genomics and Bioinformatics Research Unit, Stoneville, MS 38776}

Additional index words. DNA fingerprinting, STR markers, Chionanthus pygmaeus, Chionanthus virginicus, Osmanthus americanus, fringetree

\begin{abstract}
The genus Chionanthus (Oleaceae Hoffmans. \& Link) includes deciduous or evergreen trees and shrubs distributed widely in tropical and sub-tropical areas, including a few temperate species. Although Chionanthus species are planted as ornamental garden plants and commercialized for natural products, genetic information for Chionanthus spp. is lacking. We created microsatellite-enriched libraries of Chionanthus retusus Lindl. \& Paxton, assembled 1072 contigs, and detected 1010 repeats. The frequency of the repeats decreased with the increase in repeat length, and the most abundant motifs were: AG, AC, AAG, ACC, AT, and ACTC. We screened 384 markers on 12 accessions of four related taxa that included $C$. retusus, Chionanthus virginicus $\mathrm{L}$., Chionanthus pygmaeus Small, and Osmanthus americanus (L.) Benth. \& Hook. A total of 195 simple sequence repeat (SSR) markers amplified and discriminated six accessions of C. retusus and 57 SSR markers amplified and discriminated across the four Oleaceae species screened. To identify the best markers to use in future experiments, the "Unique Pattern Informative Combination" (UPIC) values were calculated for all the markers and the $\mathbf{1 0 0}$ markers that were most effective are reported here. The percentage of heterozygous loci across the 384 markers was lowest for $C$. retusus $(\mathbf{2 9 . 3 \% )}$ and highest for O. americanus $(\mathbf{6 8 . 9 \%})$. The SSR markers developed here could assist in taxonomy and hybridization investigations for breeding programs and authentication of varieties used as medicinal plants.
\end{abstract}

Chionanthus L. is a member of the Oleaceae, a morphologically diverse family that includes economically important genera cultivated for food (Olea europaea L.), timber (Fraxinus L.), medicinal (Forsythia Vahl.), and ornamental (Jasminum L., Ligustrum L., Osmanthus Lour., and Syringa L.) purposes. The genus Chionanthus has $\approx 100$ species (Chang et al., 1996; Wallander and Albert, 2000) distributed throughout tropical and subtropical areas worldwide but includes three temperate species (Green, 2004). Only the three temperate species are economically important as ornamentals and natural products. Temperate Chionanthus spp. (C. retusus from eastern Asia and C. pygmaeus and $C$. virginicus from eastern North America) are cultivated for their white flowers in feathery panicles and decorative blue fruits. With the exception of the pink-flowered C. pubescens Kunth from Ecuador, the evergreen tropical and subtropical Chionanthus spp. (syn Linociera Sw.) are usually not cultivated.

Very little is known about the genetics of Chionanthus. The base chromosome number in tribe Oleeae is $x=23$ with published counts for $C$. virginicus and C. retusus of $2 n=46$ (Chang et al., 1996; Taylor, 1945; Wallander and Albert, 2000). A hypothetical phylogeny for the Oleaceae, including $C$. retusus and $C$. virginicus, was developed using sequences of two noncoding chloroplast regions, the trnL-F (intron-L/spacer-F) and rps16 intron (Wallander and Albert, 2000); however, no other information at the molecular level is available that could assist in Chionanthus breeding programs or identification of species. In addition, understanding the level of genetic diversity as well as inter- and intrageneric relationships within the cultivated germplasm is important for planning a systematic breeding program for Chionanthus. Furthermore, the pollination and breeding systems of Chionanthus appear complex with individual species exhibiting varying levels of polygamodioecy (Dirr, 1998; Nicholson, 1990; Ueda, 1996) combined with occasional selfing that has implications for crossing and inheritance studies.

The development of molecular markers, specifically SSRs, will aid in assessing relationships, diversity, and parentage within the genus Chionanthus. In addition, because SSR marker transferability is usually feasible within plant families, i.e., Casuarinaceae (Yasodha et al., 2005), Meliaceae (White and Powell, 1997), Fagaceae (Barreneche et al., 2004), the markers we report here for Chionanthus could potentially transfer to species of Olea, Fraxinus, and Syringa.

\section{Materials and Methods}

Plant material. Source and accession data for all samples used in this study are shown in Table 1. Chionanthus retusus-derived SSR markers were tested on six accessions of $C$. retusus, three accessions of $C$. virginicus, two accessions of $C$. pygmaeus, and one accession of Osmanthus americanus, a species related to Chionanthus according to Wallander and Albert (2000). Total genomic DNA was extracted from leaf tissue using a Qiagen Plant Mini Kit (Qiagen, Valencia, CA).

Isolation of simple sequence repeats from Chionanthus retusus. For the construction of SSR-enriched libraries, we used the method of Techen et al. (2010), briefly described here. DNA from $C$. retusus was cut with restriction enzymes AluI, HaeIII, DraI, RsaI (New England Biolabs, Ipswich, MA) and combinations of pairs of these enzymes. The blunt-end DNA fragments were A-tailed with Taq-DNA polymerase and then ligated to an optimized 
Table 1. Chionanthus and Osmanthus samples tested with simple sequence repeats developed from Chionanthus retusus. ${ }^{\mathrm{z}}$

\begin{tabular}{lclcc}
\hline Species & Accession no. & Source and provenance & Voucher no. & Sample \\
\hline Chionanthus retusus & $37681-\mathrm{HL}$ & Kunming Inst. Bot. & 6013 & CR1 \\
Chionanthus retusus & - & Cultivated, Greenbelt, MD & 6021 & CR2 \\
Chionanthus retusus & $37681-\mathrm{HH}$ & Kunming Inst. Bot. & 6012 & CR3 \\
Chionanthus retusus & $45247-\mathrm{J}$ & Coastal Japan 1978 & 6020 & CR4 \\
Chionanthus retusus & $14089-\mathrm{L}$ & Arnold Arboretum & 6006 & CR5 \\
Chionanthus retusus & $40121-\mathrm{H}$ & Kyoto Univ. Forest Stat. & 6016 & CR6 \\
Chionanthus virginicus & - & BARC-E, Beltsville, MD & 6002 & CV8 \\
Chionanthus virginicus & - & BARC-E, Beltsville, MD & 6003 & CV9 \\
Chionanthus virginicus & $77196-\mathrm{H}$ & Shemin Nurs. & 6007 & CV10 \\
Chionanthus pygmaeus & $76993-3$ & U.S. Botanic Garden & - & CP11 \\
Chionanthus pygmaeus & $76993-2$ & U.S. Botanic Garden & - & CP12 \\
Osmanthus americanus & $56806-\mathrm{H}$ & Tom Dodd Nurs. & 6008 & OA7 \\
\hline
\end{tabular}

${ }^{z}$ All voucher specimens are located in the herbarium of the U.S. National Arboretum, Washington, DC, and are collections of J. Kirkbride and R. Olsen. Living plants of C. pygmaeus are only listed by accession number because no voucher specimens have been collected.

linker (SSRLIBF3: 5' -CGGGAGAGCAAGG AAGGAGT-3', SSRLIBR3: 5' -/5Phos/CTCC TTCCTTGCTCTCCCGAAAA'-3'). After 20 cycles of polymerase chain reaction (PCR), the amplified products were hybridized to four groups of biotinylated oligo repeats. Sequences containing repeats were captured using streptavidin-coated magnetic beads M-270 (Invitrogen, Carlsbad, CA) and the DNA was eluted. The eluate was PCR-amplified for 20 cycles; the PCR products were cloned in vector TOPO4 (Invitrogen) and sequenced using an ABI 3730XL DNA Analyzer (Applied Biosystems, Foster City, CA). Sequences were assembled in contigs using DNAStar Lasergene 7 (DNASTAR, Inc., Madison, WI) and visually checked. Repeats were searched using SSRFinder (Sharopova et al., 2002) and Sputnik (Abajian, 1994). Primers were designed using Primer3 (Rozen and Skaletsky, 2000) with stringent parameter conditions: Tm 63 optimum (60/65) minimum/maximum, length 24 optimum (20/28) minimum/maximum, and maximum overlap of repeat within the primer was $5 \mathrm{bp}$. Contig sequences containing microsatellites were screened against the NCBI Protein Database (BLASTx) (Altschul et al., 1990).

Fingerprinting. Forward primers were 5' tailed with the sequence $5^{\prime}$-CAGTTTTCCC AGTCACGAC-3' (Waldbieser et al., 2003) to permit product labeling, and reverse primers were tailed at the $5^{\prime}$ end with the sequence 5'-GTTT-3' to promote non-template adenylation (Brownstein et al., 1996). Primer 5' -CA GTTTTCCCAGTCACGAC-3' labeled with 6-carboxy-fluorescein (IDT-Technologies, Coralville, IA) was used for amplification of $10 \mathrm{ng}$ DNA using Titanium Taq DNA Polymerase (Clontech, Mountain View, CA) in $5-\mu \mathrm{L}$ reactions on an $\mathrm{M} \& \mathrm{~J}$ thermal cycler (BioRad, Hercules, CA) at $95{ }^{\circ} \mathrm{C}$ for $1 \mathrm{~min}, 60^{\circ} \mathrm{C}$ for $1 \mathrm{~min}$ (two cycles), $95^{\circ} \mathrm{C}$ for $30 \mathrm{~s}, 60^{\circ} \mathrm{C}$ for $30 \mathrm{~s}, 68^{\circ} \mathrm{C}$ for $30 \mathrm{~s}$ ( 27 cycles $)$, and a final extension at $68{ }^{\circ} \mathrm{C}$ for $4 \mathrm{~min}$. Fluorescently labeled PCR fragments were analyzed on an ABI 3730XL DNA Analyzer and dataprocessed using GeneMapper Version 3.7 (both from Applied Biosystems). Presence of alleles was converted to a binary matrix. The accessions were clustered using the unweighted paired group method and arithmetic averages (UPGMA) algorithm implemented in the SAHN program of NTSYSpc Version 2.2 (Exeter Software, Setauket, NY). The confidence level for the dendrograms was assessed by bootstrap resampling (5000 replicates) (Efron et al., 1996; Felsenstein, 1985) using WINBOOT (Yap and Nelson, 1996).

Unique Pattern Informative Combination and heterozygous loci calculations. Markers that effectively discriminated the samples tested were identified using the UPIC software (Arias et al., 2009), and the number of unique patterns (UPIC values) identified by each marker was reported for the best 100 markers. Percentage of heterozygous loci was also calculated using the UPIC software (Arias et al., 2009) for each DNA sample across all 384 SSR markers tested.

\section{Results}

Repeats found. SSR-enriched libraries of Chionanthus retusus were made using four groups of biotinylated oligo repeats. A total of 2208 clones from those libraries was sequenced. Sequences were assembled to 1072 contigs in which 1010 repeats were detected by SSRFinder and Sputnik combined. We considered repeats only those that were non-mononucleotides, had a minimum repeat length of $8 \mathrm{bp}$, and a minimum 20-bp length of flanking region upstream and downstream of the repeat. Sequences of contigs containing repeats were submitted to GenBank with accession numbers (GQ117288 to GQ118148). We designed 394 primers on the flanking regions of the repeats and tested 384 of those primers on 12 DNA samples. Primer sequences and repeat motifs as appeared in the original sequences are provided in Table 2. DNA sequences corresponding to 28 of the markers had significant hits on BLASTx indicated in Table 2 in bold. To simplify the recording of the repeat motifs, those that were circular permutations and reverse complements of each other were grouped together as one type, i.e., AAC, ACA, CAA, GTT, TGT, and TTG were recorded as AAC. This resulted in 55 non-redundant repeat motifs isolated from the C. retusus SSR-enriched libraries. The first 11 most abundant motifs detected had frequencies ranging from 560 to six as shown in Figure 1. The remaining 44 non-redundant motifs, including one CG repeat, were found in a total of 57 repeats (Table 2 ) with frequencies lower than four and were not included in the plot (data not shown). Frequencies of the isolated repeats decreased as their length increased. The number of di-, tri, tetra-, penta-, and hexanucleotides are shown in Figure 1. Only 10 of 384 markers tested did not amplify any of the 12 DNA samples used in this study.

Markers that amplified four Oleaceae species (genera Chionanthus and Osmanthus). Based on the electropherograms in GeneMapper and presence across samples, we selected 57 markers that amplified across the four species tested (C. pygmaeus, C. retusus, $C$. virginicus, and $O$. americanus). Selected primers are listed in Table 3. From those, 43 amplified all 12 DNA samples and 14 amplified nine or more DNA samples. Only two of the 57 markers were monomorphic for the four species tested; the other 55 corresponded to polymorphic loci and amplified between two and 13 alleles. A total of 350 alleles was detected by these 57 markers. From these markers, 18 were polymorphic (detected up to five alleles) within the species $C$. pygmaeus allowing distinction of the two accessions tested, whereas 35 were polymorphic (detected up to five alleles) within the species C. virginicus allowing distinction of the three accessions tested.

Because the SSRs in this study were isolated from $C$. retusus, most markers amplified predominantly this species. Of 384 markers tested, based on their ease to score, we selected 195 that amplified the six lines of C. retusus; 33 of them were monomorphic, whereas the other 162 polymorphic markers detected between two and 15 alleles. A total of 837 alleles was detected in these 195 loci within the species $C$. retusus.

Unique pattern informative combinations of markers, Unique Pattern Informative Combination values. UPIC indicates the number of DNA samples that can be discriminated by each particular marker (Arias et al., 2009). The UPIC value is more informative for selecting subsets of SSRs than the use of its polymorphism information content. We calculated UPIC values for all the markers and report the UPIC values of the best 100 in Table 3. Twelve DNA samples (Table 1) representing four species of Oleaceae (Chionanthus retusus, C. virginicus, C. pygmaeus, and Osmanthus americanus) were used for the analysis. Combinations of these markers run on the 12 DNA samples described can detect as many unique patterns or alleles as to the sum of their UPIC values. In general, the markers allowed a clear distinction among Chionanthus taxa as well as within $C$. retusus accessions, which was shown by the high bootstrap resampling coefficients obtained (Fig. 2).

Heterozygosity (\%). The percentage of heterozygous loci, based on 384 markers, for each of the four Oleaceae species tested was $29.9 \%$ to $30.7 \%$ (C. pygmaeus), $30.1 \%$ to $39.8 \%$ (C. retusus), $29.3 \%$ to $43.4 \%$ (C. virgin$i c u s)$, and $68.8 \%$ for $O$. americanus.

Cluster analysis. Genetic similarity coefficients based on UPGMA were calculated 
Table 2. Markers that amplified all the Oleaceae accessions tested detected by Unique Pattern Informative Combination software (Arias et al., 2009). ${ }^{2}$

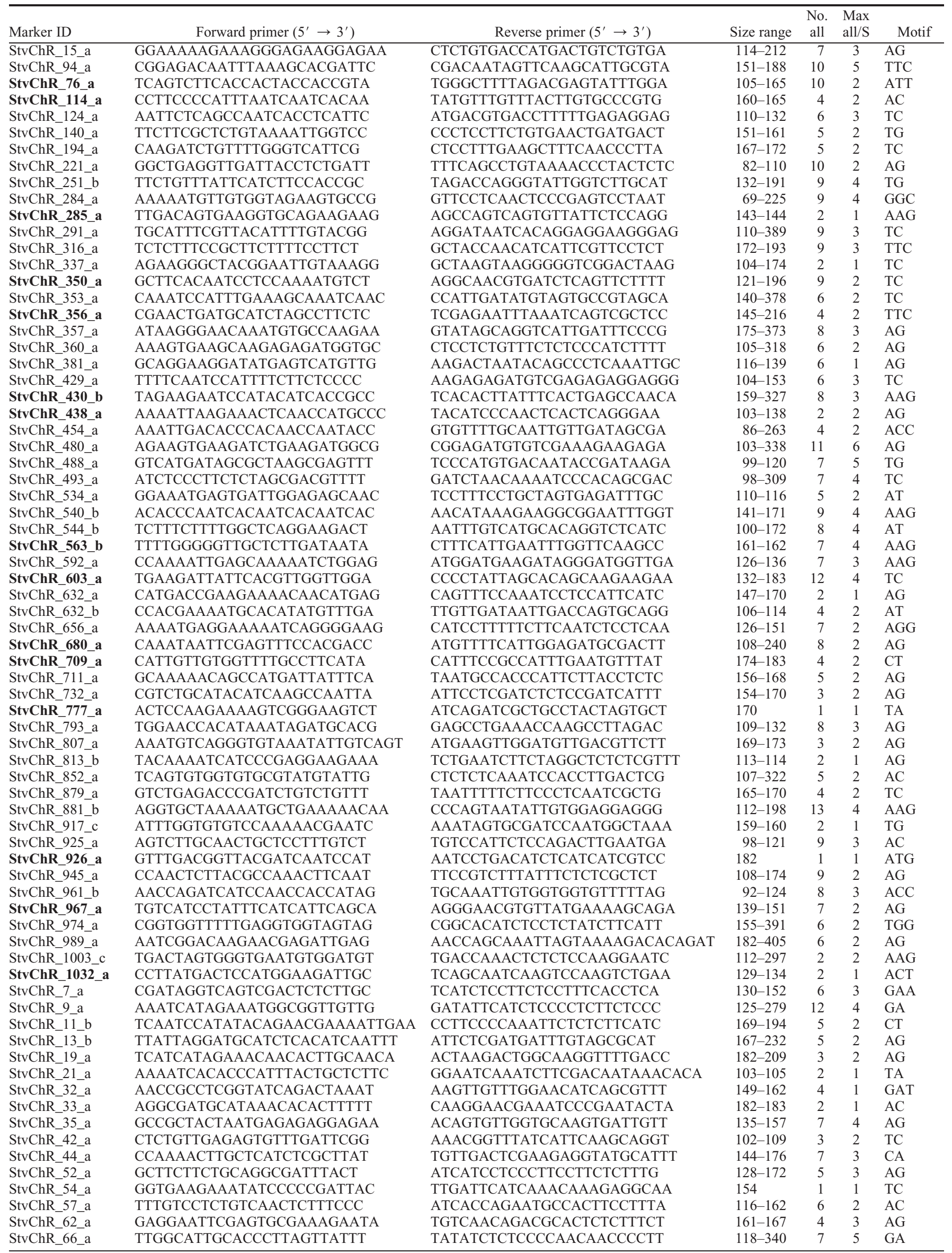




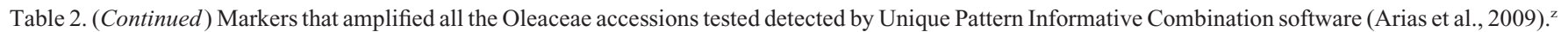

\begin{tabular}{|c|c|c|c|c|c|c|}
\hline Marker ID & Forward primer $\left(5^{\prime} \rightarrow 3^{\prime}\right)$ & Reverse primer $\left(5^{\prime} \rightarrow 3^{\prime}\right)$ & Size range & $\begin{array}{l}\text { No. } \\
\text { all }\end{array}$ & $\begin{array}{l}\text { Max } \\
\text { all/S }\end{array}$ & Motif \\
\hline StvChR_73_a & CTGGAGTGAGAGATGCAGTTCAAT & TCCCGAAACAACAGATTATTAAGGA & $181-207$ & 7 & 3 & TC \\
\hline StvChR_74_a & AGGTTTTсCтстстTTTCстAGGGTT & CGGGAATTGAAAGGAGAAAACATT & $134-144$ & 3 & 1 & GAA \\
\hline StvChR_77_a & AAACAAAGTCACCACGACCACTACT & GCGAAAAGGCTTATTGTAGAAGCA & $115-194$ & 8 & 2 & $\mathrm{ACC}$ \\
\hline StvChR_80_a & TAAAACTTGGGGCTTAAGGGGTAA & GGCTCTGACCGTCAATTGGAT & $154-164$ & 3 & 1 & $\mathrm{TC}$ \\
\hline StvChR_89_d & TTGAACTACAGATCCAATTTCCAAGA & TTTTGATCCATCCCTGTATAGCGT & $123-131$ & 4 & 2 & $\mathrm{AC}$ \\
\hline StvChR_99_a & ACTGTGTTAGCGGTGCTGTAATCA & CTCGCCATTCTCTATCTCTTCTCG & $112-154$ & 8 & 3 & $\mathrm{AG}$ \\
\hline StvChR_112_a & AATTCTTGTTTTTATTTGACGGCG & TGCAGTTGATTAAGCATGTAGCAAT & $192-260$ & 7 & 2 & AAG \\
\hline StvChR_116_a & AGGAGCGCATAGAGAGGTAGGAAT & ATCAATTCAAAATTCAAGGGCAAA & $162-166$ & 3 & 1 & $\mathrm{AG}$ \\
\hline StvChR_120_a & CCGAACCCTATTTTCTTCCATCTT & AGAGAAGGATGCGTTCACTGTTG & $107-136$ & 8 & 5 & $\mathrm{TC}$ \\
\hline StvChR_121_a & AGTGGACCATCGTCTAACTCTTCG & TTGCAGACTTTTCTTTGTTTCAACTG & $109-110$ & 2 & 1 & ATG \\
\hline StvChR_131_a & AACCAAGTCTAGATCCATTGCAGG & TCCAACGAAATGTGATGAAATGAG & $148-168$ & 7 & 3 & $\mathrm{TC}$ \\
\hline StvChR_141_a & CTTGAGTGGTTGCCCTACTTGACT & CACAGATTCAAGACACGCAGATTC & $173-184$ & 4 & 2 & $\mathrm{TC}$ \\
\hline StvChR_142_a & TGACCATTCCAACACCAAAATATG & GATCATTGCAAAAATCAGGGTTTC & $132-146$ & 4 & 2 & $\mathrm{AG}$ \\
\hline StvChR_146_a & TCCACAGATTAATGGCATTGAAAA & GAAGAAGGAGAAGGAAAAGGAGAGA & $128-131$ & 3 & 1 & $\mathrm{TC}$ \\
\hline StvChR_150_a & TAATTAATGATTGAGCGGGATTGG & AAAGTTGAGGCAATTAATGATGCTG & $269-324$ & 7 & 2 & TTC \\
\hline StvChR_159_a & TCATTTACTTTTGGTCTTCCATCAC & CCTTGTTCTTGCTGCTTGATAAAT & $165-184$ & 6 & 3 & $\mathrm{AG}$ \\
\hline StvChR_169_a & TTTGGATGTATATGGATGTGAGCG & AAGAGAGAAACAGTCCAAATCCCC & $103-283$ & 4 & 2 & $\mathrm{AG}$ \\
\hline StvChR_184_b & AAGAAGGGAAAATGTAGCGTCGTT & GTATGGGATATGTGTCGGATCCTC & $173-187$ & 5 & 2 & AAG \\
\hline StvChR_185_b & TCTGAAACCCTAACCСCTCTATCC & CGCCATCAATGTTTCCGTTATATT & $122-145$ & 5 & 2 & $\mathrm{TC}$ \\
\hline StvChR_195_a & СССTTCTATTTAGTGGGTTTGGGT & GATGAAAATGACTTCGACGATTCC & $155-169$ & 5 & 3 & $\mathrm{AG}$ \\
\hline StvChR_196_a & GCCAAACAGGGTTTTACTTTCCTT & TAAAAATGGTGAGGCAAATAACGG & $162-164$ & 2 & 2 & $\mathrm{TC}$ \\
\hline StvChR_198_a & TGCAATAGAACAGAAACAAGGCAA & TGTAGGACAGCAGTGAGTAGTCCG & $134-161$ & 10 & 4 & $\mathrm{TC}$ \\
\hline StvChR_209_b & AGAGAGAAACAAACACAAGGGCAC & GAGAAATCACTGAATAGACAGCGGA & $189-212$ & 8 & 3 & $\mathrm{TC}$ \\
\hline StvChR_227_b & CAGCCGTTGAACTTAAAGCTTCTC & CGATCCTCGCGTTTTCTATCTCTA & $155-159$ & 2 & 1 & $\mathrm{TC}$ \\
\hline StvChR_246_a & CTGTCCTGAATGCTGAATGTATGC & CCAAACCACAACAACCAACAATAC & $168-172$ & 4 & 2 & AT \\
\hline StvChR_253_a & GGAAGCAAGAAATTATTTGGGTGA & AATTATGGAGCCCAAAAAGGAAAA & $165-174$ & 3 & 2 & TG \\
\hline StvChR_254_a & CCCTAAATAACCAATAATGGATTGAAA & GTCAACCAAAACCTAGTCGTCAGA & $165-219$ & 5 & 2 & AAG \\
\hline StvChR_267_a & GGCAAATCTAATTAAATGAAAGGGTT & AACCTAAATATAACTAACGTGCACACAC & $149-468$ & 4 & 2 & ATAC \\
\hline StvChR_301_a & TGGCAATCTGAGACTTTGAGTGAG & CTACTAACCCCATCCATGCGTAAA & $125-133$ & 7 & 3 & $\mathrm{AG}$ \\
\hline StvChR_304_a & AAGATTGGACGATTCAATTTTTGTG & GAACATCGGTGACGTGGTAACTC & $104-289$ & 6 & 2 & $\mathrm{AG}$ \\
\hline StvChR_312_a & TCATTAGAGGTTGTCCAAGCCATT & TTCGAGAGGCTCGTTATTTTAGACA & $172-221$ & 7 & 2 & $\mathrm{AG}$ \\
\hline StvChR_314_a & GTAGCAAGTGAATCCAAGGAACAA & TGAAATTTTGGAACTAACTAAATCGTCT & $135-160$ & 7 & 3 & $\mathrm{AC}$ \\
\hline StvChR_318_a & GTTGTCGACGAACGTCGCTATTA & CATTTTGTCCAGCTCTACCTCCAT & $119-458$ & 4 & 2 & $\mathrm{AG}$ \\
\hline StvChR_321_b & TGATGGGGTACGAATTTTTGGTAT & ATTTTTGGAGATTTTTGGGGTGAG & $248-485$ & 7 & 4 & $\mathrm{TG}$ \\
\hline StvChR_322_a & TTTCATCGATTACAATTACCCAAATACA & TAAGATATGTGTGGGTGTTGGTGG & $111-214$ & 7 & 4 & $\mathrm{ACC}$ \\
\hline StvChR_326_b & TGACTCGTATGGAAGCAGGAAAAT & ACCGTATTCAACCACCTCAATCAT & $168-188$ & 4 & 1 & $\mathrm{TG}$ \\
\hline StvChR_327_a & GTTAGATGGGGTGAGTGAGCAAAT & TTTCATCTCCATTGGTTCCTTTCT & $159-345$ & 11 & 3 & $\mathrm{TC}$ \\
\hline StvChR_341_a & GACTTGGACTCTTGGTTGGAAAGA & GACCTCACCTTTTTGCCTCTTTTT & $123-181$ & 7 & 2 & AT \\
\hline StvChR_343_a & TTCTCTCTAATCTCGCCСCTTTCT & AGAGATGGAGGTCTGCTTCTTCAA & $157-576$ & 5 & 3 & $\mathrm{TC}$ \\
\hline StvChR_352_a & TTCGAGATATTTGAAATCTTCCTTTGA & AATGCATCAATCTCAAAGACATGC & $87-187$ & 6 & 2 & $\mathrm{TC}$ \\
\hline StvChR_371_a & GTTGACAGTGAGCGGTGTTCTCT & TTTTCTССТАТTTTСТTACCCCCG & $132-171$ & 8 & 3 & $\mathrm{AG}$ \\
\hline StvChR_379_a & AACATGCACGTCTTTACCTGTTCT & ATTTTCTTCTCCСтTCTGGGTTTC & $111-141$ & 5 & 2 & $\mathrm{TC}$ \\
\hline StvChR_411_a & GAAACTTTTCTTTGTCAGGGGTGA & TGGAAAATTTGAATTGGTAGGTGTT & $156-191$ & 4 & 1 & $\mathrm{TC}$ \\
\hline StvChR_423_a & TCTGCATAAAGCAACAATGAATCC & TGTGAGGACACTGAAAATTTGCTT & $175-184$ & 5 & 2 & $\mathrm{AG}$ \\
\hline StvChR_427_a & AGTCTTCTCTTGTCGTTCCATTCG & CTTCCACAGCACGAAAATCAGTTA & $149-153$ & 2 & 1 & $\mathrm{TC}$ \\
\hline StvChR_433_b & CСАССАТСТСААТСССАААТАСА & TATCAACATCTTCAATTGCATCGG & 175 & 1 & 1 & $\mathrm{TC}$ \\
\hline StvChR_437_a & TATAAATCGCCATTGAGGGATAGG & TCACTTTGCATAGATCAGGCGTAA & $172-176$ & 5 & 2 & $\mathrm{AG}$ \\
\hline StvChR_440_a & CACCATAATTATTGCGTTTGACCA & GGGTCACCCTCCTTTCATACTTTT & $147-187$ & 7 & 2 & $\mathrm{AC}$ \\
\hline StvChR_447_a & ATGGTCGAGATCACACAGTTGAGA & CAATTAGTCAGTAGCAGCCCCATC & $178-206$ & 6 & 2 & ATG \\
\hline StvChR_461_a & ССТCСATCССТTCTTTTGTTCTTT & TAGAGCTGTGATTGGTGAAATGGA & $151-165$ & 6 & 2 & $\mathrm{TC}$ \\
\hline StvChR_468_a & TCATAATTGACTCCATTTTCACCG & CGAGAAATGATTGAAAGTGGCTC & $102-118$ & 6 & 2 & $\mathrm{TC}$ \\
\hline StvChR_481_a & AAGATGAATTCACTCATGGGTTGC & AAGAGATTCTTTAAACCCCCACCC & $159-175$ & 5 & 2 & $\mathrm{AT}$ \\
\hline StvChR_482_a & TGAGTCATTGACAGAAGTTGAGTTTG & AGATTGAACCAAATCAAGCGATTC & $135-149$ & 4 & 2 & ATC \\
\hline StvChR_487_a & TGACCATTTGATTTAGGTGAGTGAA & TTTTGAAACCTCTACCCGATTTTT & $166-182$ & 4 & 1 & ATTT \\
\hline StvChR_490_a & CTTGTTAGGCACAAGCATTCGAT & TGTTATTACATCTTCAAACCACCATTTT & $218-224$ & 5 & 3 & $\mathrm{AG}$ \\
\hline StvChR_494_b & CTTGATTGAATTTGGTAGGATCGC & CCATAATTTCTGCACACTTGCTCA & $102-127$ & 9 & 3 & ATGT \\
\hline StvChR_496_a & TTCCTATGCСТСТСТСССТСТСТT & AGGGCGAGTTGGTGTAGACTGTAG & $152-362$ & 3 & 2 & TCC \\
\hline StvChR_504_b & ATCAATCCCACACAATTTCAAACC & AAATGAGTAGGTCGATAGGGGGAG & $96-112$ & 8 & 4 & $\mathrm{TC}$ \\
\hline StvChR_507_a & ATACTCCACCACCTGGAAATGATG & TCTCTATAGGAGGAGGAGATGGGG & $149-184$ & 9 & 4 & ATG \\
\hline StvChR_508_a & TGATGAACAATCTCGTTTATGTCCA & СТСАСССТСАТСАТСАСААСТСТС & 165 & 1 & 1 & AAG \\
\hline StvChR_510_a & GCAAGCGTATATTTTCCAACAAAA & CATGTTGAAGAATTATTTTACCCTCTTC & 99-131 & 3 & 1 & $\mathrm{AG}$ \\
\hline StvChR_515_a & TGGCTATGGTTATGGTGTTGTGTC & ACCACGCAATCTCTCCCTACATC & $143-185$ & 3 & 2 & TTG \\
\hline StvChR_525_a & GGAGAGTCTGGGGTTTATGACAGA & TTCATACCGTTTTTAACTCGTCGC & 120 & 1 & 1 & TG \\
\hline StvChR_543_a & GCGGAAGATGAAGCTATGAATGTT & TGTGTGGCTCTCATTTCACACTTT & $157-165$ & 2 & 2 & AT \\
\hline StvChR_546_a & TGGCTGGGTTTTCTTGTTACCTTA & CACACACCCATAAATTTTTCGTCA & $149-207$ & 7 & 2 & $\mathrm{AC}$ \\
\hline StvChR_566_a & TTTGAGATCTTGGTGTGGACTGTT & GATCCAAACCAGTTCTTGGAGAAA & $180-193$ & 5 & 2 & ATG \\
\hline StvChR_577_a & CATAATGCATGCACAAAACAGTCA & TTCCTGCTCCACACTACTAACATCC & $171-177$ & 3 & 2 & $\mathrm{AG}$ \\
\hline StvChR_580_a & CCCCACTTAGCATATTACATTCCA & TCGGACCTCAACATTACTCTTCAA & $120-142$ & 7 & 2 & $\mathrm{AC}$ \\
\hline StvChR_583_a & AGCAATGAGTGCTTGTAGTGTGGT & TCCAACGTTAATTATTGTCATTATCCTC & 182 & 1 & 1 & $\mathrm{AG}$ \\
\hline StvChR_587_a & CTGCAGCAGAACGGTATACACACT & AACCGTTCCCATCCTAAACTTTGT & 129 & 1 & 1 & $\mathrm{AG}$ \\
\hline StvChR_598_a & TGATTTTTGCATTTCTTGACATCTG & AAAGAAAGCGAAAGTTCAAGGACC & $123-180$ & 3 & 2 & TTC \\
\hline StvChR_604_a & CTTCTTGCTGGGACATTTTTCTTC & TGCTCTTGATTTCATTCCCAAAAT & $148-165$ & 4 & 3 & TTC \\
\hline
\end{tabular}




\begin{tabular}{|c|c|c|c|c|c|c|}
\hline Marker ID & Forward primer $\left(5^{\prime} \rightarrow 3^{\prime}\right)$ & Reverse primer $\left(5^{\prime} \rightarrow 3^{\prime}\right)$ & Size range & $\begin{array}{l}\text { No. } \\
\text { all }\end{array}$ & $\begin{array}{l}\text { Max } \\
\text { all/S }\end{array}$ & Motif \\
\hline StvChR_605_a & AATTTGAGTGACACCACACAATCG & TTCATCСТТСТТССТТСТСТСССТ & $127-541$ & 13 & 5 & TGG \\
\hline StvChR_613_a & AATGATCTCACGTAGATTCGTCCC & ATTTGGTCGGACAATGATGAGAAT & 143 & 1 & 1 & AT \\
\hline StvChR_617_a & TACCACCGCCTGGTAAATTCTC & TAAAAGTGTTGTTCGATTCGCCTT & $139-143$ & 3 & 1 & $\mathrm{ACC}$ \\
\hline StvChR_622_a & TCCAAAGCTAGAAACTCTCACAGGA & GTGGACTCAACACCACCCTTATTC & $175-194$ & 4 & 2 & $\mathrm{AG}$ \\
\hline StvChR_627_a & GGGGTGAGGGTGTTATTACTGTTG & GCCACTTCCAAATCCCACTATACA & $167-182$ & 4 & 1 & AG \\
\hline StvChR_645_c & TCAACTCAACAGGACCAAACACTG & CACAGGTGACATTTTGCCCTCTTA & 149 & 1 & 1 & AT \\
\hline StvChR_647_a & AAGCCGAAAAAGAAGAGGAAAAGA & TTAAGAATGAAGAAATGGAGCCTCA & $117-125$ & 3 & 2 & AAGG \\
\hline StvChR_655_b & ACGTAGCAGGATGAGATTCAGACA & GATTGGGTTCTGGAGTTTTGAATG & 152 & 1 & 1 & $\mathrm{AC}$ \\
\hline StvChR_659_a & TTTTATACTTGGAGGAGTGCTAATGC & GACACTATCAATCAGGGTCAAGAAAA & $149-283$ & 4 & 2 & AT \\
\hline StvChR_662_a & ACTTCAGATGTGTCATCTCTCCGA & СТАССТСТСССАСТССАССТСАТ & $178-181$ & 4 & 1 & AG \\
\hline StvChR_671_a & GAATGAAAACCTGCAAGGTCAAGT & ACCTGCAGAAAAAGCATGAGAAAA & 163 & 1 & 1 & TC \\
\hline StvChR_681_a & AATAATTTGTGATCACCACCCCAC & TGATGATCATGGAAGTGGTTATGG & 152 & 1 & 1 & $\mathrm{ACC}$ \\
\hline StvChR_686_b & GAAAGTCCGAAAGCCAGGTAATTT & GCGTATCACTGTCACCACTTCAAC & $116-126$ & 5 & 2 & $\mathrm{TC}$ \\
\hline StvChR_689_a & TGATTGATACTTTTTGATGGGGCT & TTTTCATTTTGCACTCTCСТTTCC & $175-201$ & 6 & 2 & AAG \\
\hline StvChR_697_a & CACCACCACATCCACAATGAATA & ACGATCGTGCGAATCTATGAATTT & $102-103$ & 2 & 1 & $\mathrm{ACC}$ \\
\hline StvChR_704_c & ATTTTCGAAATGTACCGGAACTGA & GTGGAGGAAGACCTCCTAGGGATA & $140-240$ & 2 & 2 & $\mathrm{TC}$ \\
\hline StvChR_730_a & TGCCTGTGATTTTGTGTATATCGTG & CCAGTGACTAGTTGAAACTTGAATTGC & $131-158$ & 6 & 2 & $\mathrm{TC}$ \\
\hline StvChR_733_a & GATCGGCTCCATTGAAAGAGAGTA & ATGCCGAGGATGATGCTGTAAT & $100-104$ & 3 & 2 & AG \\
\hline StvChR_735_a & САCTCTAACCCTAACСCСТTTGCT & GTCGAGCAAGGAACTCTACCAAAA & $121-139$ & 7 & 3 & TC \\
\hline StvChR_740_a & GTTCCTGTGATGTTCGGTTTTCTT & AGGGTGACATAATCTCCACAAGGA & $103-111$ & 4 & 3 & TG \\
\hline StvChR_752_a & GACTTTTAGAAGATTATGAGTGCCCC & TTTATTCCCATTGTTACTTTTCACAAA & $172-199$ & 7 & 3 & TC \\
\hline StvChR_753_a & TCTCGCTTCATCTTATCCTGATCC & TCAAATGTGTTATACAGGGAGGGG & $124-125$ & 2 & 1 & $\mathrm{TC}$ \\
\hline StvChR_759_b & TAACACGGACACATCCTAAGCAAC & TGTGTCTCTGAAATGGTTGGTTGT & $126-143$ & 5 & 2 & $\mathrm{ACC}$ \\
\hline StvChR_786_a & GATTAACAAAATCATTCCTCCCCC & CATGCAAACATGTTCTATGTGCAG & $108-118$ & 6 & 2 & $\mathrm{TC}$ \\
\hline StvChR_795_a & TCATCTACСТCTGAACATAATTCCCA & GGCTGTATTGGCTTAGTGAAATGG & $140-176$ & 7 & 3 & AACC \\
\hline StvChR_808_a & GAGCAAAGGTAAACAAGAAGCGAA & GTCGATTTCTTCCTCGATGTCTGT & 163 & 1 & 1 & $\mathrm{AG}$ \\
\hline StvChR_825_a & TTGGATTAATTTTGGTTTGTTTATTGTT & TGGAAGAGAGTTTGTGCAAGGTAG & $172-192$ & 5 & 2 & TC \\
\hline StvChR_832_b & TGTTTTATTGTGCAAAAATCCGGT & GGTGGAGAATGAGGAGAGAAGTGA & $158-185$ & 7 & 3 & $\mathrm{TC}$ \\
\hline StvChR_836_c & CACCAGATCAACAAATCCCATACC & GTGTGATAGATGTGGTGGTTGCAG & $100-218$ & 14 & 5 & ACCATC \\
\hline StvChR_858_a & TTTACCGTGAGAGATAATGGGAGC & TTAGTTGAGTGACTTGACCACCGA & $165-203$ & 6 & 2 & $\mathrm{AG}$ \\
\hline StvChR_862_a & GCTTTTAAGAAACTACGTAAGATGGGG & GCATTTGATTTCTTAAGGTCACTCG & $159-179$ & 3 & 2 & $\mathrm{AG}$ \\
\hline StvChR_865_a & GTCTCTTCAGGTTTACTCGACCCC & TTGACTGTGAAGTCGTGGGCT & 136 & 1 & 1 & ATC \\
\hline StvChR_894_a & TGAACATTCACTAATCAGCCTCCTC & AGAGAAGAAGACGGACCGGTAGAC & $178-358$ & 5 & 3 & $\mathrm{TC}$ \\
\hline StvChR_896_a & GTCCGGATTCACTTTCTTCCTTTC & ATGATAATGGCGATGGTCTCGTAA & $98-119$ & 4 & 2 & TTC \\
\hline StvChR_918_c & AAAATCCTCCACATTCAACCAGAA & CGACGTTCACTGTTCGTTCACT & $173-184$ & 3 & 2 & $\mathrm{AC}$ \\
\hline StvChR_927_a & TAAAATCCGACTTTTCCCTCACAA & CACCAACTTTGGCTAAGAAAAATGA & 172 & 1 & 1 & $\mathrm{TC}$ \\
\hline StvChR_935_a & TAAGCCTTGAAGTAGTAGCTGCCC & ATCGAACTTAAAGTGCTGCCAATC & $83-189$ & 5 & 2 & TC \\
\hline StvChR_943_a & TTACTCCAATCGCTTTCCTAAACG & CAGCCTACATCCAAACTTTGTTCC & $123-146$ & 2 & 2 & AG \\
\hline StvChR_950_a & TATTACCCTATTCCCTGACGAGCA & GGGAAGGAGAAAAACAAAGAGGAA & $155-181$ & 5 & 2 & TG \\
\hline StvChR_976_a & TACGTAGGGAGGAATGTTCAAACG & GCAAGGAACATCAAAAGTCCATCT & 179 & 1 & 1 & AAG \\
\hline StvChR_996_a & ATTTGAGGGGAATAGGGAAATCGT & AAGTCGAAGACTCGAAGTCCCAC & 99-134 & 5 & 3 & TG \\
\hline StvChR_1006_a & TGCTGACCACGTTGTTATCTGTTT & ACTGACGTGGAACCAAGAAAGAAG & $97-113$ & 3 & 3 & $\mathrm{TC}$ \\
\hline StvChR_1016_a & GCTTTCCTTTTTGTTTCСстстTC & TTCATCCAAAGATTTCGAGTGTAAAA & $155-183$ & 5 & 2 & TTC \\
\hline StvChR_1017_a & GACCAACCAAAACAATCCAACTC & CTCCGGTGGTAAATTGAAAGAGAG & $92-127$ & 15 & 5 & $\mathrm{TC}$ \\
\hline StvChR_1025_a & AGCCCATTTTTGCATTTTGAGTAT & ACAATGATTTTGTGTTTTGATTTGTCT & $157-182$ & 4 & 1 & $\mathrm{AC}$ \\
\hline StvChR_1031_b & TTTGAATCCATCCATTTGAGAACA & TGGGAGAAAAATGAGACTTGGTTT & $139-146$ & 8 & 4 & $\mathrm{AC}$ \\
\hline StvChR_1061_a & CACAATTGCACCCAATTCCTACAT & ССАТССТССТАААСССТСАТТТТС & 150 & 1 & 1 & AT \\
\hline StvChR_1072_a & TACCAGAGATGGGAACAACACAGA & TTTTAGGAGGGGTGAAATTGATGA & $146-161$ & 5 & 4 & $\mathrm{AAG}$ \\
\hline StvChR_217_sk/a & ACTTGAAGATTTATCGGCACATCC & GATGGACCTTTCCATGTTCTTGAC & $147-169$ & 5 & 3 & $\mathrm{TC}$ \\
\hline StvChR_351_sk/a & AGTTTTCTCTCTTTACTCACGCCACT & AATGAAGGCGACAATTCCACTAAA & $154-184$ & 6 & 3 & ACTC \\
\hline StvChR_432_sk/a & GTCTCCGACGATGAACCAAATACT & TTTCAAACCTTTGTTCTCGTCTCC & $165-176$ & 2 & 2 & $\mathrm{AG}$ \\
\hline StvChR_568_sk/a & TCCCAAAATACCCAAAGAAAACAA & TTGGAATCCTTTTCTCCTATTCCC & $149-150$ & 2 & 1 & AAGAT \\
\hline StvChR_710_sk/a & GTAGAAGAAGGGGAAATGGGAAGA & CTATCCTGGGTGGGTATGAAATTG & $174-191$ & 5 & 2 & AAGAG \\
\hline StvChR_745_sk/a & CACACTCCATCGCAACAATAGAAG & TGTGTGGTTGATTAGTTCGTGGTT & $99-157$ & 8 & 3 & AACC \\
\hline StvChR_955_sk/a & ATTTCCCGCCCATACACATACAC & CGACCGAGTCCTCCATAGAGATTT & $172-217$ & 7 & 2 & $\mathrm{AC}$ \\
\hline
\end{tabular}

${ }^{2}$ Markers that amplified all the Oleaceae (StvChR_15_a to StvChR_1032_a) and Chionanthus retusus only (StvChR_7_a to StvChR_955_sk/a). DNA sequences shown in bold had significant hits on BLASTx. In the columns, size range is: observed on the samples, includes the 23 bp of primer tailing. "No. all" $=$ total number of alleles detected. "Max all/S" = maximum number of alleles detected on an individual sample. Sequences corresponding to the contigs were submitted to GenBank with accession numbers GQ117288 to GQ118.

for four species of Oleaceae (C. pygmaeus, $C$. retusus, $C$. virginicus, and $O$. americanus) using 57 markers as shown in Figure 2A. We also calculated genetic similarity coefficients for $C$. retusus using 195 markers as shown in Figure 2B. We observed a high level of polymorphism within the species $C$. retusus using these 195 markers. These markers allowed a clear distinction among $C$. retusus varieties as is shown by the high bootstrap resampling coefficients obtained. Bootstrap confidence values are indicated on the dendrograms. Clusters formed groups by species with the exception of $C$. virginicus (6007), which associated with $C$. pygmaeus based on the 57 markers analyzed as shown in Figure 2A.

\section{Discussion}

Chionanthus SSR-enriched libraries were generated using 24 oligo repeats; however, we detected twice as many repeat motifs, probably as a result of non-specific hybridization during the enrichment process. Although we detected a large number of repeats in the assembled contigs, only 394 pairs of primers were designed under the stringent conditions used. In C. retusus, the frequency of repeats decreased with the increase in length of the repeat motifs (Fig. 1). This trend has also been observed in the distribution of SSRs of other eukaryotic genomes (Katti et al., 2001). We also found that in $C$. retusus, the most abundant di-, tri, and tetranucleotide repeat motives were $\mathrm{AG} / \mathrm{AC}$, AAG/ACC, and ACTC/ATAC, respectively. In Arabidopsis thaliana (L.) Heynh., the same di- and trinucleotide motives were the most abundant across the entire genome; 

motives were of the type "AAA/(CTG)" (Anwar and Khan, 2005). Curiously, we found at least one $(\mathrm{CG})_{\mathrm{n}}$ repeat in $C$. retusus, a particular repeat motif that has not been found in the entire $A$. thaliana genome (Anwar and Khan, 2005) and that is very rare in other eukaryotic genomes (Katti et al., 2001).

Ninety-six percent of 57 SSRs that amplified across the four species of Oleaceae $(C$. pygmaeus, $C$. retusus, $C$. virginicus, and $O$. americanus) were polymorphic. Within the species C. retusus, $61 \%$ of 195 SSRs we developed were polymorphic. The high level ples of $C$. retusus that covered two extremes of its range in Asia with C. retusus CR1 and CR3 originating from the Yunnan province in China and CR4 and CR6 from Japan. Chionanthus retusus CR2 represents the typical however, the predominant tetranucleotide of polymorphism is not unexpected in this study, because we chose representative sam-

form in cultivation in the United States and is likely derived from the same parental stock as CR5, a plant we originally received as $C$. retusus var. serrulatus (Hayata) Koidz. from the Arnold Arboretum, but is of uncertain provenance. The varietal epithet serrulatus was first applied to a new species of Chionanthus from Taiwan, which was later reduced to a variety of C. retusus (Fogg, 1960). A larger survey of North American nurseries is planned to determine the level of genetic diversity of C. retusus in cultivation. Only a handful of $C$. virginicus and $C$. pygmaeus accessions were available for sampling and used to test crossamplification of $C$. retusus-derived SSRs. A broader sampling, encompassing a greater range of $C$. virginicus in eastern North America and larger sampling in Florida, where it is in close proximity to $C$. pygmaeus, is needed to determine population structure and similarities between these species.

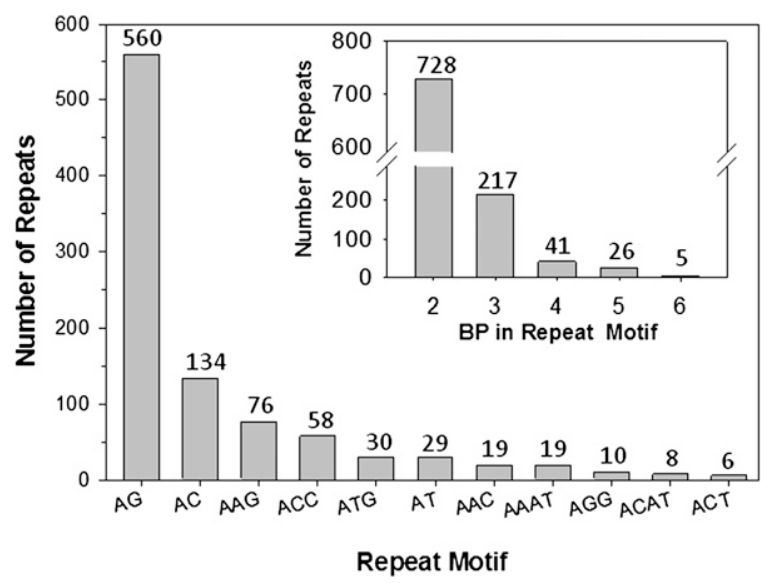

Fig. 1. Motifs and frequency of repeats detected in Chionanthus retusus simple sequence repeat (SSR)enriched libraries. Frequency of motif repeats detected in an SSR-enriched library after screening 1072 contigs. Another 43 repeat motifs with frequencies lower than four were not included in the plot. Embedded graph shows the number of repeats detected in C. retusus arranged by repeat motif length (BP).
The percentage of heterozygous loci found in $O$. americanus $(68.8 \%)$ was twofold higher than in C. retusus (30.1\% to $39.8 \%$ ). In addition, $O$. americanus shared $29 \%$ of detected alleles with $C$. retusus, which had a genetic similarity of 0.54 . Very few alleles were shared between $O$. americanus and the species $C$. virginicus and $C$. pygmaeus. The amplification and sharing of alleles between Chionanthus sp. and $O$. americanus is indicative of a recent divergence and supports the conclusions of Wallander and Albert (2000) who reported $O$. americanus to be closely related to Chionanthus. The greater number of polymorphic loci in $O$. americanus may also be attributed to genome size. Whereas Chionanthus species used in this study are presumed diploid with $2 n=46$, Osmanthus americanus is a hexaploid with $2 n=138$ (Taylor, 1945).

BLASTx screening of DNA sequences containing microsatellites showed significant hits on some interesting genes. For example, the sequence that originated marker StvCHR_508a had similarity to SORBIDRAFT of Sorghum bicolor that is related to the development of inflorescences (Polegri et al., 2010). The DNA sequence corresponding to marker StvCHR_680a had similarity to proteasome subunit alpha; both $26 \mathrm{~S}$ proteasome and the REV (REVOLUTA) genes are required for the maintenance of root apical meristem and shoot apical meristem (Zhang et al., 2010), and it has been shown that plants with double mutations on both isoforms of the $26 \mathrm{~S}$ proteasome are often non-viable (Gallois et al., 2009). Several DNA sequences had similarity to chromatin remodeling, DNA binding, and control of transcription such as those from markers StvCHR_356a, StvCHR_430b, StvCHR_777a, and StvCHR 943a. The DNA sequences associated to marker StvCHR_709a had similarity to the brahma-related gene (BRG1), which is implicated in tumor suppressor function (Hendricks et al., 2004). Further research taking in

Table 3. Markers that amplified DNA samples from four species of Oleaceae (Chionanthus retusus, C. virginicus, C. pygmaeus, and Osmanthus americanus) selected by their performance in terms of peak quality and distribution across species.

\begin{tabular}{|c|c|c|c|c|c|c|c|c|c|}
\hline Marker & UPIC & Marker & UPIC & Marker & UPIC & Marker & UPIC & Marker & UPIC \\
\hline StvChR_945a & 12 & StvChR_209b & 6 & StvChR_793a & 5 & StvChR_656a & 4 & StvChR_447a & 4 \\
\hline StvChR_350a & 10 & StvChR_120a & 6 & StvChR_343a & 5 & StvChR_740a & 4 & StvChR_430b & 4 \\
\hline StvChR_57a & 10 & StvChR_504b & 6 & StvChR_321b & 5 & StvChR_832b & 4 & StvChR_124a & 4 \\
\hline StvChR_35a & 10 & StvChR_494b & 6 & StvChR_185b & 5 & StvChR_896a & 4 & StvChR_195a & 4 \\
\hline StvChR_9a & 8 & StvChR_881b & 6 & StvChR_689a & 5 & StvChR_351sk & 4 & StvChR_1017a & 4 \\
\hline StvChR_540b & 8 & StvChR_301a & 6 & StvChR_76a & 5 & StvChR_141a & 4 & StvChR_437a & 4 \\
\hline StvChR_925a & 8 & StvChR_131a & 6 & StvChR_352a & 5 & StvChR_99a & 4 & StvChR_312a & 4 \\
\hline StvChR_112a & 8 & StvChR_221a & 6 & StvChR_546a & 4 & StvChR_786a & 4 & StvChR_44a & 4 \\
\hline StvChR_11b & 8 & StvChR_955sk & 6 & StvChR_440a & 4 & StvChR_825a & 4 & StvChR_989a & 3 \\
\hline StvChR_66a & 8 & StvChR_198a & 6 & StvChR_184b & 4 & StvChR_314a & 4 & StvChR_304a & 3 \\
\hline StvChR_62a & 8 & StvChR_490a & 6 & StvChR_468a & 4 & StvChR_745sk & 4 & StvChR_411a & 3 \\
\hline StvChR_251b & 7 & StvChR_935a & 6 & StvChR_961b & 4 & StvChR_894a & 4 & StvChR_480a & 3 \\
\hline StvChR_603a & 7 & StvChR_150a & 6 & StvChR_73a & 4 & StvChR_680a & 4 & StvChR_563b & 3 \\
\hline StvChR_566a & 7 & StvChR_461a & 6 & StvChR_284a & 4 & StvChR_254a & 4 & StvChR_1025a & 3 \\
\hline StvChR_316a & 7 & StvChR_580a & 6 & StvChR_94a & 4 & StvChR_1006a & 4 & StvChR_33b & 3 \\
\hline StvChR_19a & 7 & StvChR_605a & 6 & StvChR_1016a & 4 & StvChR_481a & 4 & StvChR_598a & 3 \\
\hline StvChR_795a & 6 & StvChR_13b & 6 & StvChR_967a & 4 & StvChR_327a & 4 & StvChR_711a & 3 \\
\hline StvChR_836c & 6 & StvChR_371a & 6 & StvChR_507a & 4 & StvChR_159a & 4 & StvChR_77a & 3 \\
\hline StvChR_710sk & 6 & StvChR_169a & 5 & StvChR_752a & 4 & StvChR_15a & 4 & StvChR_544b & 3 \\
\hline StvChR_341a & 6 & StvChR_357a & 5 & StvChR_730a & 4 & StvChR_950a & 4 & StvChR_326b & 3 \\
\hline
\end{tabular}

UPIC values correspond to the number of DNA samples that were discriminated by the marker out of the 12 lines tested from Table 1 . Combinations of these markers can detect a number of unique patterns or alleles equal to the sum of their UPIC values.

UPIC = Unique Pattern Informative Combination. 

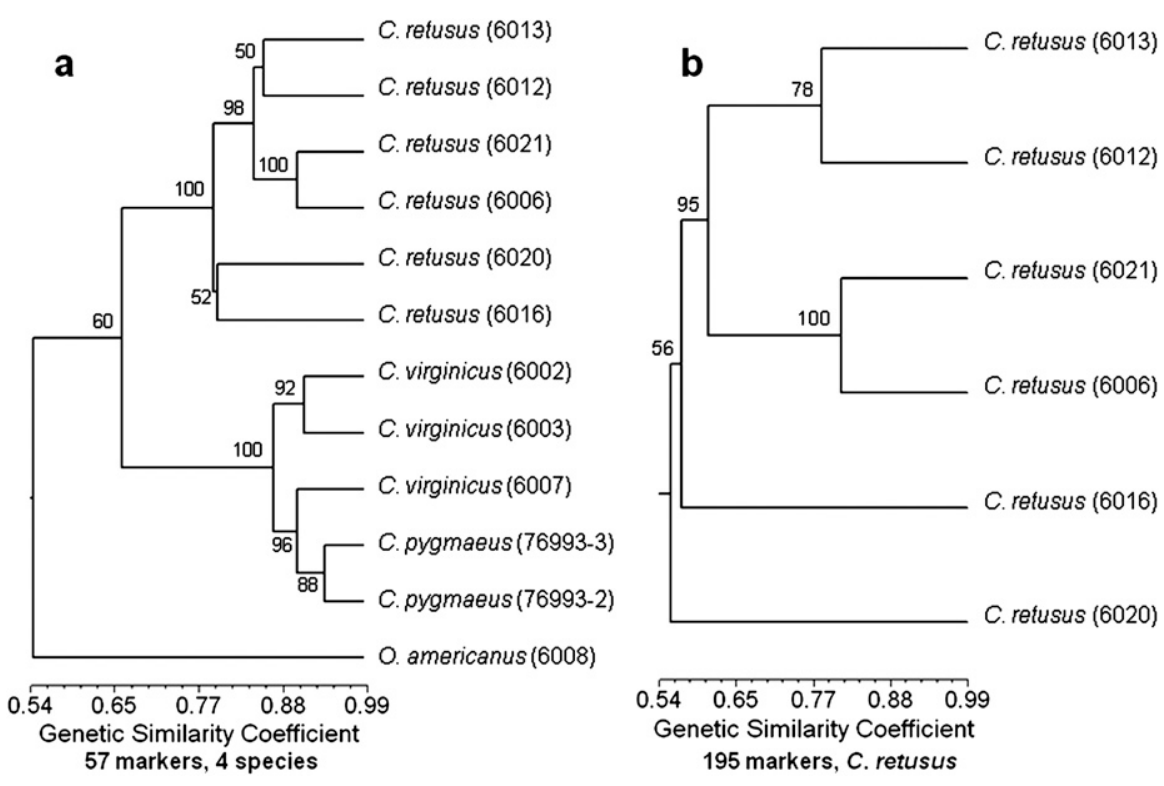

Fig. 2. Clusters calculated using the unweighted paired group method using arithmetic averages (UPGMA) of NTSYSpc 2.2. Confidence levels from bootstrap analysis (5000 replicates) are indicated at the nodes. (A) Cluster analysis of 12 DNA samples of Chionanthus and Osmanthus using 57 simple sequence repeat (SSR) markers that amplified across species; (B) cluster analysis of 6 DNA samples of C. retusus using 195 SSR markers that amplified this species.

consideration the gene functions potentially affected by the polymorphism of the markers presented here would provide useful insight for plant breeding programs.

Considering that transferability of molecular markers is feasible within plant families (Barreneche et al., 2004; White and Powell, 1997; Yasodha et al., 2005), the markers we developed for Chionanthus could be used to examine other economically important members of the Oleaceae such as Fraxinus and Olea. Although a number of microsatellites are available for Olea (Cipriani et al., 2002; Omrani-Sabbaghi et al., 2007; Stambuk et al., 2007), between 200 and 500 additional markers are necessary for $80 \%$ to $95 \%$ of the Olea genome to lie within $10 \mathrm{cM}$ of a marker (Wu et al., 2004).

In the present study, we have characterized a large number of SSR markers for Chionanthus-related species: 55 that discriminate among the four species of Oleaceae tested, 162 that discriminate within the species $C$. retusus, 35 polymorphic ones for $C$. virginicus, and 18 polymorphic markers for $C$. pygmaeus. These markers could aid in identifying genetic diversity of Chionanthus germplasm and allow verification of hybrids, pedigrees, and cultivars for botanical characterization and ornamental tree breeding programs.

\section{Literature Cited}

Abajian, C. 1994. Sputnik. Aug. 2010. <http:// espressosoftware.com/sputnik/index.html $>$.

Altschul, S.F., W. Gish, W. Miller, E.W. Myers, and D.J. Lipman. 1990. Basic local alignment search tool. J. Mol. Biol. 215:403-410.

Anwar, T. and A.U. Khan. 2005. Mapping and analysis of simple sequence repeats in the Arabidopsis thaliana genome. Bioinformation 1:64-68.
Arias, R.S., L.E. Ballard, and B.E. Scheffler. 2009. UPIC: Perl scripts to determine the number of SSR markers to run. Bioinformation 3:353361.

Barreneche, T., M. Casasoli, K. Russell, A. Akkak, H. Meddour, C. Plomion, F. Villani, and A. Kremer. 2004. Comparative mapping between Quercus and Castanea using simple-sequence repeats (SSRs). Theor. Appl. Genet. 108:558566.

Brownstein, M.J., J.D. Carpten, and J.R. Smith. 1996. Modulation of non-templated nucleotide addition by Taq DNA polymerase: Primer modifications that facilitate genotyping. Biotechniques 20:1004-1006, 1008-1010.

Chang, M.-C., L.C. Chiu, Z. Wei, and P.S. Green. 1996. Oleaceae: Chionanthus, p. 293-295. In: $\mathrm{Wu}, \mathrm{Z}$. and P.H. Raven (eds.). Flora of China. Vol. 15. Science Press, Beijing, China.

Cipriani, G., M.T. Marrazzo, R. Marconi, A Cimato, and R. Testolin. 2002. Microsatellite markers isolated in olive (Olea europaea L.) are suitable for individual fingerprinting and reveal polymorphism within ancient cultivars. Theor. Appl. Genet. 104:223-228.

Dirr, M.A. 1998. Manual of woody landscape plants. Stipes Publishing, Champaign, IL.

Efron, B., E. Halloran, and S. Holmes. 1996. Bootstrap confidence levels for phylogenetic trees. Proc. Natl. Acad. Sci. USA 93:13429-13434.

Felsenstein, J. 1985. Confidence-limits on phylogenies-An approach using the bootstrap. Evolution 39:783-791.

Fogg, J.M.J. 1960. Chionanthus in the Philadelphia area. Morris Arboretum Bul. 11:3-6.

Gallois, J.L., A. Guyon-Debast, A. Lecureuil, D. Vezon, V. Carpentier, S. Bonhomme, and P. Guerche. 2009. The arabidopsis proteasome RPT5 subunits are essential for gametophyte development and show accession-dependent redundancy. Plant Cell 21:442-459.

Green, P.S. 2004. Oleaceae, p. 296-306. In: Kubitzki, K. (ed.). The families and genera of vascular plants. 7th Ed. Springer-Verlag, Berlin, Germany.
Hendricks, K.B., F. Shanahanand, and E. Lees. 2004. Role for BRG1 in cell cycle control and tumor suppression. Mol. Cell. Biol. 24:362376.

Katti, M.V., P.K. Ranjekar, and V.S. Gupta. 2001. Differential distribution of simple sequence repeats in eukaryotic genome sequences. Mol. Biol. Evol. 18:1161-1167.

Nicholson, R.G. 1990. The fringe tree and its farflung cousins. Arnoldia 50:24-31.

Omrani-Sabbaghi, A., M. Shahriari, M. FalahatiAnbaran, S.A. Mohammadib, A. Nankalic, M. Mardia, and B. Ghareyazie. 2007. Microsatellite markers based assessment of genetic diversity in Iranian olive (Olea europaea L.) collections. Sci. Hort. 112:439-447.

Polegri, L., O. Calderini, S. Arcioni, and F. Pupilli. 2010. Specific expression of apomixis-linked alleles revealed by comparative transcriptomic analysis of sexual and apomictic Paspalum simplex Morong flowers. J. Expt. Bot. 61: 1869-1883.

Rozen, S. and H. Skaletsky. 2000. Bioinformatics methods and protocols. In: Methods in molecular biology Totowa, NJ.

Sharopova, N., M.D. McMullen, L. Schultz, et al. 2002. Development and mapping of SSR markers for maize. Plant Mol. Biol. 48:463481.

Stambuk, S., D. Sutlovic, P. Bakaric, S. Petricevic, and S. Andelinovic. 2007. Forensic botany: Potential usefulness of microsatellite-based genotyping of Croatian olive (Olea europaea L.) in forensic casework. Croat. Med. J. 48: 556-562.

Taylor, H. 1945. Cyto-taxonomy and phylogeny of the Oleaceae. Brittonia 5:337-367.

Techen, N., R.S. Arias, Z. Pan, I. Khan, and B.E. Scheffler. 2010. Optimized construction of microsatellite-enriched libraries. Mol. Ecol. Resources 10:508-515.

Ueda, K. 1996. Androdioecism in Chionanthus retusus (Oleaceae). J. Phytotax. Taxon. 44:9192.

Waldbieser, G.C., S.M. Quiniou, and A. Karsi. 2003. Rapid development of gene-tagged microsatellite markers from bacterial artificial chromosome clones using anchored TAA repeat primers. Biotechniques 35:976-979.

Wallander, E. and V.A. Albert. 2000. Phylogeny and classification of Oleaceae based on rps16 and trnL-F sequence data. Amer. J. Bot. 87: 1827-1841.

White, G. and W. Powell. 1997. Cross-species amplification of SSR loci in the Meliaceae family. Mol. Ecol. 6:1195-1197.

Wu, S.B., G. Collins, and M. Sedgley. 2004. A molecular linkage map of olive (Olea europaea L) based on RAPD, microsatellite, and SCAR markers. Genome 47:26-35.

Yap, I.V. and R.J. Nelson. 1996. Winboot: A program for performing bootstrap analysis of binary data to determine the confidence limits of UPGMA based dendrograms. IRRI Discussion Paper Series No. 14. International Rice Research Institute, Manila. May 2009. <http:/ www.irri.org/science/software>.

Yasodha, R., M. Ghosh, R. Sumathi, and K. Gurumurthi. 2005. Cross-species amplification of eucalyptus SSR markers in Casuarinaceae. Acta Botanica Croatica 64:115-120.

Zhang, Z., H. Wang, D. Luo, M. Zeng, H. Huang, and X. Cui. 2010. Convergence of the 26S proteasome and the REVOLUTA pathways in regulating inflorescence and floral meristem functions in Arabidopsis. J. Expt. Bot. doi: 10. 1093/jxb/erq277. 